\title{
ON THE HAUSDORFF DIMENSION FAITHFULNESS AND THE CANTOR SERIES EXPANSION
}

\author{
S. ALBEVERIO, GANNA IVANENKO, MYKOLA LEBID, AND GRYGORIY TORBIN
}

\begin{abstract}
We study families $\Phi$ of coverings which are faithful for the Hausdorff dimension calculation on a given set $E$ (i. e., special relatively narrow families of coverings leading to the classical Hausdorff dimension of an arbitrary subset of $E$ ) and which are natural generalizations of comparable net-coverings. They are shown to be very useful for the determination or estimation of the Hausdorff dimension of sets and probability measures. We give general necessary and sufficient conditions for a covering family to be faithful and new techniques for proving faithfulness/non-faithfulness for the family of cylinders generated by expansions of real numbers. Motivated by applications in the multifractal analysis of infinite Bernoulli convolutions, we study in details the Cantor series expansion and prove necessary and sufficient conditions for the corresponding net-coverings to be faithful. To the best of our knowledge this is the first known sharp condition of the faithfulness for a class of covering families containing both faithful and non-faithful ones.

Applying our results, we characterize fine fractal properties of probability measures with independent digits of the Cantor series expansion and show that a class of faithful net-coverings essentially wider that the class of comparable ones. We construct, in particular, rather simple examples of faithful families $\mathcal{A}$ of net-coverings which are "extremely non-comparable" to the Hausdorff measure.
\end{abstract}

Ми досліджуємо сім'ї $\Phi$ покриттів, які є довірчими для обчислення розмірності Хаусдорфа-Безиковича на певній множині $E$ (тобто, спеціальні відносно вузькі сім'ї покриттів, яких достатньо для коректного обчислення класичної розмірності Хаусдорфа-Безиковича довільної підмножини множини $E$ ) і які є природним узагальненням порівнянних мережевих покриттів. В роботі показано, що такі сім’і $є$ дуже корисними для обчислення чи оцінки розмірності Хаусдорфа-Безиковича множин та ймовірнісних мір.

Нами отримано загальні необхідні та достатні умови довірчості для сімей покриттів та запропоновано нову техніку доведення довірчості/недовірчості для сімей циліндрів, породжених різними розкладами дійсних чисел. Маючи додаткову мотивацію в мультифрактальному аналізі нескінченних згорток Бернуллі, ми детально дослідили розклади Кантора та довели необхідні та достатні умови довірчості відповідних сімей покриттів мережевими циліндрами. Наскільки нам відомо, ці результати є першими критеріями довірчості для класу сімей покриттів, що містить як довірчі, так і недовірчі сім'ї.

Застосовуючи отримані результати, ми дослідили тонкі фрактальні властивості ймовірнісних мір з незалежними символами розкладів Кантора і показали, що клас довірчих мережевих покриттів суттєво ширше за клас порівнянних. Ми побудували, зокрема, досить прості приклади довірчих сімей $\mathcal{A}$ мережевих покриттів, які є "екстремально непорівнянними" відносно міри Хаусдорфа.

\section{INTRODUCTION}

The notion of the Hausdorff dimension is well-known now and is of great importance in mathematics as well as in diverse applied problems (see, e.g., [12, 23, 28, 36, 45]). In many situations the determination (or even estimations) of this dimension for sets

2020 Mathematics Subject Classification. 11K55, 28A80, 60G30.

Keywords. fractals, Hausdorff dimension, faithful and non-faithful covering families, Cantor series expansion, comparable net measures, infinite Bernoulli convolutions, singular probability measures. 
from a given family or even for a given set is a rather non-trivial problem (see, e. g., $[1,8,13,17,23,32,37]$ and references therein). Different approaches and special methods for the determination of the Hausdorff dimension are collected in [23, 24, 31]. A new approach based on the theory of transformations preserving the Hausdorff dimension (DP-transformations) was presented in [7, 9] and has been developed in [4, 26, 27]. In this paper we develop another approach which is deeply connected with the theory of DP-transformations as well as with the following well known approach: to simplify the calculation of the Hausdorff dimension of a given set it is extremely useful to have an appropriate and a relatively narrow family of admissible coverings which lead to the same value of the dimension. We shall start to deal with one-dimensional sets and show later how our results can be naturally extended to the multidiminsional case and to the general case of metric spaces.

Without loss of generality we shall consider subsets from the unit interval. Let $\Phi$ be a fine family of coverings on $[0,1]$, i.e., a family of subsets of $[0,1]$ such that for any $\varepsilon>0$ there exists an at most countable $\varepsilon$ - covering $\left\{E_{j}\right\}$ of $[0,1]$ with $E_{j} \in \Phi$. Let us shortly recall that the $\alpha$-dimensional Hausdorff measure of a set $E \subset[0,1]$ w. r. t. a given fine family of coverings $\Phi$ is defined by

$$
H^{\alpha}(E, \Phi)=\lim _{\varepsilon \rightarrow 0}\left[\inf _{\left|E_{j}\right| \leq \varepsilon}\left\{\sum_{j}\left|E_{j}\right|^{\alpha}\right\}\right]=\lim _{\varepsilon \rightarrow 0} H_{\varepsilon}^{\alpha}(E, \Phi),
$$

where the infimum is taken over all at most countable $\varepsilon$-coverings $\left\{E_{j}\right\}$ of $E, E_{j} \in \Phi$.

We remark that, generally speaking, $H^{\alpha}(E, \Phi)$ depends on the family $\Phi$. The family of all subsets of $[0,1]$ and the family of all closed (open) subintervals of $[0,1]$ give rise to the same $\alpha$-dimensional Hausdorff measure, which will be denoted by $H^{\alpha}(E)$. The nonnegative number

$$
\operatorname{dim}_{H}(E, \Phi)=\inf \left\{\alpha: H^{\alpha}(E, \Phi)=0\right\}
$$

is called the Hausdorff dimension of the set $E \subset[0,1]$ w. r. t. a family $\Phi$. If $\Phi$ is the family of all subsets of $[0,1]$, or $\Phi$ coincides with the family of all closed (open) subintervals

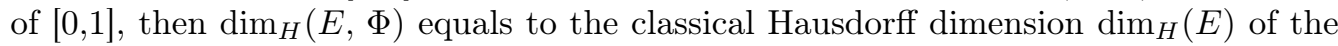
subset $E \subset[0,1]$.

The notion of comparable net measures are also well known now (see, e. g. , [22, 40]). Roughly speaking, net measures are special cases of $H^{\alpha}(E, \Phi)$, where the family $\Phi$ consists of sets with the following properties: 1) if $A_{1}$ and $A_{2}$ belong to $\Phi$, then $A_{1} \subset A_{2}$ or $A_{2} \subset A_{1}$ or $\left.A_{1} \bigcap A_{2}=\emptyset ; 2\right) \Phi$ is countable; 3) at most a finite number of sets from $\Phi$ contain any given set from $\Phi$. Then the corresponding net measure $H^{\alpha}(E, \Phi)$ is said to be comparable to Hausdorff measure if the ratios of measures are bounded above and below. It has been shown that comparable net measures are very useful in the study of Hausdorff measures (see, e.g., [15, 22, 30, 40] and references therein).

In this paper we actually develop theory of measures which are generalizations of comparable net measures in the following sense.

Definition. A fine covering family $\Phi$ is said to be faithful family of coverings (non-faithful family of coverings) for the Hausdorff dimension calculation on $[0,1]$ if

$$
\begin{gathered}
\operatorname{dim}_{H}(E, \Phi)=\operatorname{dim}_{H}(E), \quad \forall E \subseteq[0,1] \\
\text { (resp. } \left.\exists E \subseteq[0,1]: \operatorname{dim}_{H}(E, \Phi) \neq \operatorname{dim}_{H}(E)\right) .
\end{gathered}
$$

It is clear that any family $\Phi$ of comparable net-coverings (i.e., net-coverings which generate comparable net-measures) is faithful. Conditions for a fine covering family to be faithful were studied by many authors (see, e.g., [11, 16, 20, 39] and references therein). First steps in this direction have been done by A. Besicovitch ([15]), who proved the 
faithfulness for the family of cylinders of binary expansion. His result was extended by $\mathrm{P}$. Billingsley ([16]) to the family of $s$-adic cylinders, by M. Pratsiovytyi ([44]) to the family of $Q$-S-cylinders, and by S. Albeverio and G. Torbin ([11]) to the family of $Q^{*}$-cylinders for those matrices $Q^{*}$ whose elements $p_{0 k}, p_{(s-1) k}$ are bounded from zero. Some general sufficient conditions for the faithfulness of a given family of coverings are also known ([20], [39]). Let us mentioned here that all these results were obtained by using the standard approach: if for a given family $\Phi$ there exist positive constants $\beta \in \mathbb{R}$ and $N^{*} \in \mathbb{N}$ such that for any interval $B=(a, b)$ there exist at most $N^{*}$ sets $B_{j} \in \Phi$ which cover $(a, b)$ and $\left|B_{j}\right| \leq \beta \cdot|B|$, then the family $\Phi$ is faithful. It is clear that all above mentioned families of net-coverings are even comparable. The faithfulness of underlying families of basic cylinders plays a crucial role in the studying of fractal properties of non-normal as well as essentially non-normal numbers in different systems of numerations (see, e.g., $[1,4,5,6,38])$ and fine fractal properties of singularly continuous probability measures (see, e.g., $[1,11,32]$ and references therein).

It is rather paradoxical that initial examples of non-faithful families of coverings appeared firstly in two-dimensional case (as a result of active studies of self-affine sets during the last decade of XX century (see, e.g., [14])). The family of cylinders of the classical continued fraction expansion can probably be considered as the first (and rather unexpected) example of non-faithful one-dimensional net-family of coverings ([35]). By using approach, which has been invented by Yuval Peres to prove non-faithfulness of the family of continued fraction cylinders ([35]), in [2] authors have proven the non-faithfulness for the family of cylinders of $Q_{\infty}$-expansion with polynomially decreasing elements $\left\{q_{i}\right\}$. The latter two families of coverings give examples of non-comparable net measures.

So, it is natural to ask about the existence of faithful covering families which are not comparable.

We study this problem and give general necessary and sufficient conditions for a fine covering system to be faithful. The main aim of the paper is to study faithful properties of the covering families which are generated by the famous Cantor series expansions. Let us recall that for a given sequence $\left\{n_{k}\right\}_{k=1}^{\infty}$ with $n_{k} \in \mathbb{N} \backslash\{1\}, k \in \mathbb{N}$ the expression of $x \in[0,1]$ in the following form

$$
x=\sum_{k=1}^{\infty} \frac{\alpha_{k}}{n_{1} \cdot n_{2} \cdot \ldots \cdot n_{k}}=: \Delta_{\alpha_{1} \alpha_{2} \ldots \alpha_{k} \ldots,}, \alpha_{k} \in\left\{0,1, \ldots, n_{k}-1\right\}
$$

is said to be the Cantor series expansion of $x$. These expansions, which have been initially studied by G. Cantor in 1869 (see., e.g. [18]), are natural generalizations of the classical $s$-adic expansion for reals. Cantor series expansions have been intensively studied from different points of view during last century (see, e.g., [21, 29, 41, 25] and references therein). Our own motivations to study faithful properties of such expansions came from our investigations on fine fractal properties of infinite Bernoulli convolutions, i.e., probability distributions of the following random variables

$$
\xi=\sum_{k=1}^{\infty} \xi_{k} a_{k}
$$

where $\sum_{k=1}^{\infty} a_{k}$ is a convergent positive series, and $\xi_{k}$ are independent random variables taking values 0 and 1 with probabilities $p_{0 k}$ and $p_{1 k}$ respectively. Measures of this form have been studied since 1930's from the pure probabilistic point of view as well as for their applications in harmonic analysis, dynamical systems and fractal analysis [33]. The Lebesgue structure and fine fractal properties of the distribution of $\xi$ are well studied for the case where $r_{k}:=\sum_{i=k+1}^{\infty} a_{i} \geq a_{k}$ for all large enough $k$ (see, e.g., [10, 19]). The 
case where $a_{k}<r_{k}$ holds for an infinite number of $k$ can be considered as a «Terra incognita» in this field. Even for the case where $a_{k}=\lambda^{k}$ and $p_{0 k}=\frac{1}{2}$ the problem of singularity is still open ([42]). Main problems here are related to the fact that almost all points from the spectrum of $\xi$ have uncountably many different expansion in the form $\sum \varepsilon_{k} a_{k}, \varepsilon_{k} \in\{0,1\}$. This is the so-called «Bernoulli convolutions with large overlaps». We consider two special classes of such measures. The first one is generated by sequences $a_{k}$ with the following properties: $\exists\left\{m_{k}\right\}$ such that $r_{m_{k}}>a_{m_{k}}=a_{m_{k}+1}+\ldots+a_{m_{k}+s_{k}}$, $s_{k}=m_{k+1}-m_{k}-1 \geq 2, \quad k \in N, r_{j}=a_{j}$, for $j \notin\left\{m_{k}\right\}$.

The second one is connected to the sequences $a_{k}$ such that $\forall k \in N \quad \exists s_{k} \in N \bigcup\{0\}$ : $a_{k}=a_{k+1}=\ldots=a_{k+s_{k}}=r_{k+s_{k}}$, and $s_{k}>0$ for an infinite number of indices $k$. In both cases singularity plays a generic role and to study fine fractal properties of the corresponding probability distributions it is necessary to have knowledge on faithfulness (non-faithfulness) of fine families of partitions which are turned to be the Cantor series partitions.

Main result of the present paper states that the family $\mathcal{A}$ of Cantor coverings of the unit interval is faithful for the Hausdorff dimension calculation if and only if

$$
\lim _{k \rightarrow \infty} \frac{\ln n_{k}}{\ln n_{1} \cdot n_{2} \cdot \ldots \cdot n_{k-1}}=0 .
$$

To the best of our knowledge this theorem gives the first necessary and sufficient condition of the faithfulness for a class of covering families containing both faithful and non-faithful ones. The proof of this result is given in the next Section. As a corollary of our results we characterize fine fractal properties of probability measures with independent digits of the Cantor series expansion and show that a class of faithful net-coverings essentially wider that the class of comparable net-coverings. We construct, in particular, simple examples of faithful families $\mathcal{A}$ of net-coverings which are "extremely" non-comparable to the Hausdorff measure.

\section{Sharp CONDitions FOR THE HAUSDORFF Dimension FAithFulness OF THE CANTOR SERIES EXPANSION}

In this Section we give some general conditions for a fine covering family to be faithful for the Hausdorff dimension calculation and prove necessary and sufficient conditions for the Cantor series net-coverings to be faithful.

We start firstly with a very useful lemma, which can be proven easily, and, nevertheless, presents general necessary and sufficient conditions for the faithfulness.

Lemma 2.1. Let $\Phi$ be a fine covering family on $[0,1]$. Then $\Phi$ is faithful on the unit interval if and only if there exists a positive constant $C$ such that for any $E \subset[0,1]$, any $\alpha \in(0,1]$ and any $\delta \in(0, \alpha)$ the following inequality holds:

$$
H^{\alpha}(E, \Phi) \leq C \cdot H^{\alpha-\delta}(E) .
$$

Let us mention that this lemma can be obviously generalized to a multidimensional Euclidean space and even to any metric space, which can be equipped by fine covering families.

Based on the latter lemma one can easily get the following sufficient condition for the faithfulness of a fine covering family.

Lemma 2.2. Let $\Phi$ be a fine covering family of $[0,1]$. Assume that there exists a positive constant $C$ and a function $N(x): R_{+} \rightarrow \mathbb{N}$ such that:

1) for any interval $I \subset[0,1]$ there exist at most $N(|I|)$ subsets

$$
\triangle_{1}^{I}, \triangle_{2}^{I}, \ldots, \triangle_{l(I)}^{I} \in \Phi
$$


with

$$
l(I) \leq N(|I|),\left|\triangle_{j}^{I}\right| \leq|I| \text { and } I \subset \bigcup_{j=1}^{l(I)} \triangle_{j}^{I} ;
$$

2) for any $\delta>0$ there exists $\varepsilon_{1}(\delta)>0$ such that

$$
N(|I|) \cdot|I|^{\delta} \leq C, \forall I \subset[0,1] \text { with }|I|<\varepsilon_{1}(\delta) .
$$

Then the family $\Phi$ is faithful on $[0,1]$.

Let $\left\{n_{k}\right\}_{k=1}^{\infty}$ be a sequence with $n_{k} \in \mathbb{N} \backslash\{1\}, k \in \mathbb{N}$. Let us recall that the expression of $x$ in the following form

$$
x=\sum_{k=1}^{\infty} \frac{\alpha_{k}}{n_{1} \cdot n_{2} \cdot \ldots \cdot n_{k}}=: \Delta_{\alpha_{1} \alpha_{2} \ldots \alpha_{k} \ldots,}, \alpha_{k} \in\left\{0,1, \ldots, n_{k}-1\right\}
$$

is said to be the Cantor series expansion of a real number $x \in[0,1]$.

For a given sequence $\left\{n_{k}\right\}_{k=1}^{\infty}$ let $\mathcal{A}_{k}$ be the family of the k-th rank intervals (cylinders) , i.e.,

$$
\mathcal{A}_{k}:=\left\{E: E=\Delta_{\alpha_{1} \alpha_{2} \ldots \alpha_{k}}, \alpha_{i} \in \overline{1, n_{i}}, i=1,2, \ldots, k\right\} .
$$

Let $\mathcal{A}$ be the family of all possible rank intervals, i.e.,

$$
\mathcal{A}:=\left\{E: E=\Delta_{\alpha_{1} \alpha_{2} \ldots \alpha_{k}}, n \in \mathbb{N}, \alpha_{i} \in \overline{1, n_{i}}, i=1,2, \ldots, n\right\},
$$

which is said to be the Cantor covering family.

The following theorem gives necessary and sufficient conditions for a Cantor covering family to be faithful.

Theorem 2.3. The family $\mathcal{A}$ of Cantor coverings of the unit interval is faithful for the Hausdorff dimension calculation if and only if

$$
\lim _{k \rightarrow \infty} \frac{\ln n_{k}}{\ln n_{1} \cdot n_{2} \cdot \ldots \cdot n_{k-1}}=0 .
$$

Proof. Sufficiency. Let (2.4) holds. It is enough to prove that

$$
\operatorname{dim}_{H}(E) \geq \operatorname{dim}_{H}(E, \Phi), \forall E \subset[0,1] .
$$

Let $I$ be an arbitrary interval. Then there exists an interval $\Delta(k(I))=\Delta_{\alpha_{1} \ldots \alpha_{k(I)}} \in \mathcal{A}$ such that:

1) $\Delta_{\alpha_{1} \ldots \alpha_{k(I)}} \subset I$

2) any interval of $(k(I)-1)$ th rank does not belong to $I$.

The interval $I$ contains at most $2 \cdot n_{k(I)}$ intervals from $\mathcal{A}_{k}$. So $I$ can be covered by $N(|I|)=2 \cdot n_{k(I)}+2$ intervals from $\mathcal{A}_{k}$. Therefore,

$$
|\Delta(k(I))| \leq|I|<N(|I|) \cdot|\Delta(k(I))| \cdot
$$

Let $C$ be an arbitrary positive constant. Then the equality

$$
\lim _{k \rightarrow \infty} \frac{\ln n_{k}}{\sum_{i=1}^{k-1} \ln n_{i}}=0
$$

holds if and only if for any positive $\delta$ there exists $k_{0}(\delta) \in \mathbb{N}$ such that $\forall k>k_{0}(\delta)$ :

$$
\left(2 \cdot n_{k}+2\right) \cdot\left(\frac{2 \cdot n_{k}+2}{n_{1} \cdot n_{2} \cdot \ldots \cdot n_{k-1} \cdot n_{k}}\right)^{\delta} \leq C .
$$

Therefore $\forall \delta>0, \exists k_{0}(\delta), \forall k>k_{0}(\delta)$ :

$$
N(|I|) \cdot|I|^{\delta} \leq C .
$$

So, from Lemma 2.2 it follows that $\mathcal{A}$ is faithful for the Hausdorff dimension calculation. 
Necessity. Now we show that if

$$
\varlimsup_{k \rightarrow \infty} \frac{\ln n_{k}}{\ln n_{1} \cdot n_{2} \cdot \ldots \cdot n_{k-1}}=: C>0,
$$

then $\mathcal{A}$ is non-faithful for the Hausdorff dimension calculation. To this end we shall construct a set $T=T(C)$ with the following properties:

$$
\begin{aligned}
\text { 1) } \operatorname{dim}_{H}(T) & \leq \frac{2}{2+C} ; \\
\text { 2) } \operatorname{dim}_{H}(T, \mathcal{A}) & \geq \frac{4+C}{4+3 C} .
\end{aligned}
$$

From (2.5) it follows that there exists a subsequence $\left\{k_{i}\right\}$ such that $\forall \delta \in(0, C), \exists N_{0}(\delta)$, $\forall k_{i}>N_{0}(\delta)$ :

$$
\left(n_{1} n_{2} \ldots n_{k_{i}-1}\right)^{C-\delta} \leq n_{k_{i}} \leq\left(n_{1} n_{2} \ldots n_{k_{i}-1}\right)^{C+\delta} .
$$

It is clear that $\forall \varepsilon>0, \exists N_{1}(\varepsilon): \forall k>N_{1}(\varepsilon)$ :

$$
\frac{1}{n_{1} \cdot n_{2} \cdot \ldots \cdot n_{k-1}}<\varepsilon
$$

Let $N_{2}(\varepsilon, \delta):=\max \left\{N_{0}(\delta), N_{1}(\varepsilon)\right\}$. Let us choose a subsequence $\left\{k_{j}^{\prime}\right\}$ from the sequence $\left\{k_{i}\right\}$ with the following property:

$$
\frac{\ln \left(n_{k_{j-1}^{\prime}+1} \ldots n_{k_{j}^{\prime}-1}\right)}{\ln \left(n_{1} n_{2} \ldots n_{k_{j-1}^{\prime}-1} n_{k_{j-1}^{\prime}} n_{k_{j-1}^{\prime}+1} \ldots n_{k_{j}^{\prime}-1}\right)}>1-\frac{C}{4},
$$

and construct the set $T$ in the following way:

$$
\begin{aligned}
T=\left\{x: x \in[0,1), x=\sum_{k=1}^{\infty} \frac{\alpha_{k}(x)}{\prod_{i=1}^{k} n_{i}}, \alpha_{k}(x) \in \overline{0,\left[\sqrt{n_{k}}\right]}\right. \\
\left.\quad \text { if } k \in\left\{k_{j}^{\prime}\right\}, \text { and } \alpha_{k}(x) \in \overline{0, n_{k}-1} \text { if } k \notin\left\{k_{j}^{\prime}\right\}\right\} .
\end{aligned}
$$

Firstly let us show that

$$
\operatorname{dim}_{H}(T) \leq \frac{2}{2+C}
$$

Let $k_{j}^{\prime}>N_{2}(\varepsilon, \delta)$. The set $T$ can be covered by $n_{1} \cdot n_{2} \cdot \ldots \cdot n_{k_{j}^{\prime}-1}$ intervals and each of them is a union of $\left[\sqrt{n_{k_{j}^{\prime}}}\right]+1$ sets from $\mathcal{A}_{k_{j}^{\prime}}$. The $\alpha$-volume of this $\varepsilon$-covering is equal to

$$
n_{1} n_{2} \ldots n_{k_{j}^{\prime}-1}\left(\frac{\left[\sqrt{n_{k_{j}^{\prime}}}\right]+1}{n_{1} n_{2} \ldots n_{k_{j}^{\prime}}}\right)^{\alpha} .
$$

From (2.6) it follows that

Suppose

$$
n_{1} n_{2} \ldots n_{k_{j}^{\prime}-1}\left(\frac{\left[\sqrt{n_{k_{j}^{\prime}}}\right]+1}{n_{1} n_{2} \ldots n_{k_{j}^{\prime}}}\right)^{\alpha} \leq 2^{\alpha}\left(n_{1} n_{2} \ldots n_{k_{j}^{\prime}-1}\right)^{1-\frac{1}{2} \alpha(C-\delta)-\alpha},
$$

then

$$
1-\frac{1}{2} \alpha(C-\delta)-\alpha<0
$$

$$
H_{\varepsilon}^{\alpha}(T) \leq \lim _{j \rightarrow \infty} 2^{\alpha}\left(n_{1} n_{2} \ldots n_{k_{j}^{\prime}-1}\right)^{1-\frac{1}{2} \alpha(C-\delta)-\alpha}=0 .
$$


Therefore,

So,

$$
H_{\varepsilon}^{\alpha}(T)=0, \forall \alpha>\frac{2}{C-\delta+2}, \forall \varepsilon>0, \forall \delta>0
$$

and, hence,

$$
\operatorname{dim}_{H} T \leq \frac{2}{C-\delta+2}, \forall \delta>0
$$

Now let us show that

$$
\operatorname{dim}_{H} T \leq \frac{2}{C+2}
$$

Let

$$
\operatorname{dim}_{H}(T, \mathcal{A}) \geq \frac{4+C}{4+3 C} .
$$

$$
\left\{k_{j}^{\prime \prime}\right\}=\left\{k_{j}^{\prime}\right\} \bigcap\left\{N_{2}(\varepsilon, \delta)+1, N_{2}(\varepsilon, \delta)+2, \ldots\right\} .
$$

Let $\mu=\mu_{N_{2}(\varepsilon, \delta)}$ be the probability measure corresponding to the random variable

$$
\xi=\sum_{k=1}^{\infty} \frac{\xi_{k}}{\prod_{i=1}^{k} n_{i}}
$$

where $\xi_{k}$ are independent random variables; if $k \in\left\{k_{j}^{\prime \prime}\right\}$, then $\xi_{k}$ takes values $0,1, \ldots,\left[\sqrt{n_{k}}\right]$ with probabilities $\frac{1}{\left[\sqrt{n_{k}}\right]+1}$; if $k \notin\left\{k_{j}^{\prime \prime}\right\}$, then $\xi_{k}$ takes values $0,1, \ldots, n_{k}-1$ with probabilities $\frac{1}{n_{k}}$.

Then

for any $\Delta_{\alpha_{1} \alpha_{2} \ldots \alpha_{k}}$ from $\mathcal{A}_{k}$, and

$$
\left|\Delta_{\alpha_{1} \alpha_{2} \ldots \alpha_{k}}\right|=\frac{1}{n_{1} n_{2} \ldots n_{k}}
$$

$$
\mu\left(\Delta_{\alpha_{1} \alpha_{2} \ldots \alpha_{k}}\right)=\frac{1}{\varphi_{1} \varphi_{2} \ldots \varphi_{k}}
$$

where $\varphi_{t}=n_{t}$ if $t \notin\left\{k_{j}^{\prime \prime}\right\}$ and $\varphi_{t}=\left[\sqrt{n_{t}}\right]+1$ if $t \in\left\{k_{j}^{\prime \prime}\right\}, \forall t \in \mathbb{N}$.

Let us show that

$$
\frac{\ln \left(\mu\left(\Delta_{\alpha_{1} \alpha_{2} \ldots \alpha_{k}}\right)\right)}{\ln \left(\left|\Delta_{\alpha_{1} \alpha_{2} \ldots \alpha_{k}}\right|\right)} \geq \frac{4+C-2 \delta}{4+3 C+4 \delta}, \forall k \in \mathbb{N} .
$$

Taking into account properties of $\left\{k_{j}^{\prime \prime}\right\}$, one can prove by induction on $j$ that

$$
\frac{\ln \left(\varphi_{1} \varphi_{2} \ldots \varphi_{k_{j}^{\prime \prime}}\right)}{\ln \left(n_{1} n_{2} n_{3} \ldots n_{k_{j}^{\prime \prime}}\right)} \geq \frac{4+C-2 \delta}{4+3 C+4 \delta}, \forall j \in \mathbb{N}
$$

Let $k \in\left(k_{j}^{\prime \prime}, k_{j+1}^{\prime \prime}\right)$. Then

$$
\frac{\ln \left(\mu\left(\Delta_{\alpha_{1} \alpha_{2} \ldots \alpha_{k}}\right)\right)}{\ln \left(\left|\Delta_{\alpha_{1} \alpha_{2} \ldots \alpha_{k}}\right|\right)} \geq \frac{\ln \left(\mu\left(\Delta_{\alpha_{1} \alpha_{2} \ldots \alpha_{k_{j}^{\prime \prime}}}\right)\right)}{\ln \left(\left|\Delta_{\alpha_{1} \alpha_{2} \ldots \alpha_{k_{j}^{\prime \prime}}}\right|\right)} \geq \frac{4+C-2 \delta}{4+3 C+4 \delta}, \forall k \in \mathbb{N} .
$$

Let $\left\{\Delta_{i}^{\prime}\right\}$ be an arbitrary $\varepsilon$ - covering of $\mathrm{T}, \Delta_{i}^{\prime} \in \mathcal{A}, \forall i \in \mathbb{N}$. Then, using (2.11) we get

$$
\frac{4+C-2 \delta}{4+3 C+4 \delta} \leq \frac{\ln \left(\mu\left(\Delta_{i}^{\prime}\right)\right)}{\ln \left(\left|\Delta_{i}^{\prime}\right|\right)}<1
$$

which implies that

$$
\mu\left(\Delta_{i}^{\prime}\right) \leq\left|\Delta_{i}^{\prime}\right|^{\frac{4+C-2 \delta}{4+3 C+4 \delta}}
$$


Let $\alpha \in\left[0, \frac{4+C-2 \delta}{4+3 C+4 \delta}\right)$. Then we have

$$
1=\mu(T) \leq \bigcup_{i} \mu\left(\Delta_{i}^{\prime}\right) \leq \sum_{i}\left|\Delta_{i}^{\prime}\right|^{\frac{4+C-2 \delta}{4+3 C+4 \delta}} \leq \sum_{i}\left|\Delta_{i}^{\prime}\right|^{\alpha} .
$$

So, $\forall \delta>0, \forall \varepsilon>0, \alpha \in\left[0, \frac{4+C-2 \delta}{4+3 C+4 \delta}\right)$, and for any $\varepsilon$ - covering $\left\{\Delta_{i}^{\prime}\right\}$ of the set $T$ by cylinders $\Delta_{i}^{\prime} \in \mathcal{A}$ we have

$$
\sum_{i}\left|\Delta_{i}^{\prime}\right|^{\alpha} \geq 1
$$

Therefore,

$$
H_{\varepsilon}^{\alpha}(T, \mathcal{A}) \geq 1, \quad \forall \delta>0, \forall \varepsilon>0, \alpha \in\left[0, \frac{4+C-2 \delta}{4+3 C+4 \delta}\right) .
$$

So,

$$
H^{\alpha}(T, \mathcal{A}) \geq 1, \quad \forall \delta>0, \forall \alpha<\frac{4+C-2 \delta}{4+3 C+4 \delta}
$$

Hence,

$$
\operatorname{dim}_{H}(T, \mathcal{A}) \geq \frac{4+C-2 \delta}{4+3 C+4 \delta}, \forall \delta>0
$$

and, therefore,

$$
\operatorname{dim}_{H}(T, \mathcal{A}) \geq \frac{4+C}{4+3 C}
$$

which completes the proof.

\section{Some APPLiCATIONS}

First application of this theorem will be connected with fine fractal properties of random Cantor expansions. Let us recall (see, e.g., [43]) that for a given probability measure $\mu$ the number

$$
\operatorname{dim}_{H} \mu=\inf \left\{\operatorname{dim}_{H}(E): \mu(E)=1\right\}
$$

is said to be the Hausdorff dimension of the measure $\mu$. In the case of singularity this number is a rather important characteristic of a probability measure (see, e.g., [11]) Applying the latter theorem and methods from [11], we get the Hausdorff dimension of the probability distribution $\mu_{\xi}$ of the random variable $\xi$ with independent digits of the Cantor series expansion, i.e.,

$$
\xi=\sum_{k=1}^{\infty} \frac{\xi_{k}}{n_{1} n_{2} \ldots n_{k}},
$$

where independent $\xi_{k}$ take values $0,1, \ldots, n_{k}-1$ with probabilities $p_{0 k}, p_{1 k}, \ldots, p_{n_{k}-1, k}$ respectively.

Proposition 3.1. Let $h_{j}=-\sum_{i=0}^{n_{k}-1} p_{i j} \ln p_{i j}$ and $H_{k}=\sum_{j=1}^{k} h_{j}$. If

$$
\sum_{k=1}^{\infty}\left(\frac{\ln n_{k}}{\ln \left(n_{1} n_{2} \ldots n_{k}\right)}\right)^{2}<\infty,
$$

then the Hausdorff dimension of the probability distribution $\mu_{\xi}$ of the random variable $\xi$ with independent digits of the Cantor series expansion is equal to

$$
\operatorname{dim}_{H}\left(\mu_{\xi}\right)=\lim _{k \rightarrow \infty} \frac{H_{k}}{\ln \left(n_{1} n_{2} \ldots n_{k}\right)} .
$$


Now let us consider examples which show essential differences between the notions of faithful net-coverings and comparable net-coverings.

Example 1. Let $n_{k}=4^{k}, \forall k \in \mathbb{N}$ and let $\mathcal{A}$ be the net-covering family generated by the corresponding Cantor series expansion. Let

$$
A=\left\{x: x \in[0,1], x=\sum_{k=1}^{\infty} \frac{\alpha_{k}(x)}{\prod_{i=1}^{k} n_{i}}, \alpha_{k}(x) \in\left\{0,1, \ldots, 2^{k}-1\right\}, \quad \forall k \in \mathbb{N}\right\} .
$$

Then

1) $\operatorname{dim}_{H} A=\frac{1}{2}$;

2) $H^{\frac{1}{2}}(A, \mathcal{A}) \geq 1$

3) $H^{\frac{1}{2}}(A)=0$.

Proof. Let $\lambda$ be Lebesgue measure on the unit interval and let $\mu_{\xi}$ be the probability measure of the random variable

$$
\xi=\sum_{k=1}^{\infty} \frac{\xi_{k}}{\prod_{i=1}^{k} n_{i}}
$$

where $\xi_{k}$ are independent random variables taking values $0,1, \ldots, 2^{k}-1$ with probabilities $\frac{1}{2^{k}}$. Let $\Delta_{n}(x)$ be the $\mathrm{n}$-th rank cylinder of the Cantor series expansion containing $x$. It is clear that for any $x \in A$ one has

$$
\mu_{\xi}\left(\Delta_{n}(x)\right)=2^{-\frac{n(n+1)}{2}} \quad \text { and } \quad \lambda\left(\Delta_{n}(x)\right)=4^{-\frac{n(n+1)}{2}} .
$$

So,

$$
\frac{\ln \mu_{\xi}\left(\Delta_{n}(x)\right)}{\ln \lambda\left(\Delta_{n}(x)\right)}=\frac{1}{2}, \quad \forall x \in A
$$

Using the Theorem 2.5 from [16], we get $\operatorname{dim}_{H}(A, \mathcal{A})=\frac{1}{2}$. From our theorem one can obviously derive the faithfulness of the family $\mathcal{A}$ for the case $n_{k}=4^{k}$. Therefore $\operatorname{dim}_{H}(A)=\frac{1}{2}$.

Let $\left\{E_{j}\right\}$ be an arbitrary $\varepsilon$-covering of the set $A$ by cylinders from $\mathcal{A}$. Without loss of generality we may assume that $E_{j} \cap A \neq \emptyset$, i.e., $E_{j}=\Delta_{n_{j}}(x)$ for some $x \in A$. Applying the mass distributional principle, we have

$$
1=\mu(A)=\mu\left(\bigcup_{j} E_{j}\right) \leq \sum_{j} \mu\left(E_{j}\right)=\sum_{j}\left|E_{j}\right|^{\frac{1}{2}}
$$

for any $\varepsilon$-covering of $A$ by cylinders from $\mathcal{A}$. Therefore, $H^{\frac{1}{2}}(A, \mathcal{A}) \geq 1$.

The set $A$ can be covered by $2^{1} \cdot 2^{2} \cdot \ldots \cdot 2^{k-1} \cdot 1$ intervals (each of them is a union of $2^{k} \mathrm{k}$-th rank cylinders) with length $2^{-k^{2}}$. The $\frac{1}{2}$-volume of this covering is equal to $2^{\frac{(k-1) k}{2}} \cdot\left(2^{-k^{2}}\right)^{\frac{1}{2}}$, which tends to 0 as $k \rightarrow \infty$. Therefore, $H^{\frac{1}{2}}(A)=0$.

The following example shows that a faithful net-covering family can be "extremely non-comparable" to the Hausdorff measure.

Example 2. Let $n_{k}=4^{k}$ and let $\mathcal{A}$ be the net-covering family generated by the corresponding Cantor series expansion. Let

$$
\begin{gathered}
T=\left\{x: x \in[0,1], x=\sum_{k=1}^{\infty} \frac{\alpha_{k}(x)}{\prod_{i=1}^{k} 4^{i}} \text { with } \alpha_{k}(x) \in \overline{0, \sqrt{n_{k}}-1} \text { if } k \neq 2^{s},\right. \\
\left.\alpha_{k}(x) \in \overline{0, k \cdot \sqrt{n_{k}}-1} \text { if } k=2^{s}, s \in \mathbb{N}\right\} .
\end{gathered}
$$

Then the family $\mathcal{A}$ is faithful for the Hausdorff dimension calculation and 
1) $\operatorname{dim}_{H} T=\frac{1}{2}$;

2) $H^{\frac{1}{2}}(T, \mathcal{A})=+\infty$

3) $H^{\frac{1}{2}}(T)=0$.

Proof. Let $\mu_{\xi}$ be the probability measure with respect to the random variable

$$
\xi=\sum_{k=1}^{\infty} \frac{\xi_{k}}{\prod_{i=1}^{k} n_{i}},
$$

where $\xi_{k}$ are independent random variables with following distributions:

if $k \neq 2^{s}$, then $\xi_{k}$ takes values $0,1, \ldots, 2^{k}-1$ with probabilities $\frac{1}{2^{k}}$;

if $k=2^{s}$, then $\xi_{k}$ takes values $0,1, \ldots, k \cdot 2^{k}-1$ with probabilities $\frac{1}{k \cdot 2^{k}}$.

Let $\Delta_{n}(x)$ be the $\mathrm{n}$-th rank cylinder of the Cantor series expansion containing $x$. From the construction of $\xi$ it follows that for any $x \in T$ one has

$$
\mu_{\xi}\left(\Delta_{n}(x)\right)=2^{-\left(\frac{n(n+1)}{2}+\frac{\left(\left[\log _{2} n\right]+1\right)\left[\log _{2} n\right]}{2}\right)} \text { and } \lambda\left(\Delta_{n}(x)\right)=4^{-\frac{n(n+1)}{2}} .
$$

So,

$$
\lim _{n \rightarrow \infty} \frac{\ln \mu_{\xi}\left(\Delta_{n}(x)\right)}{\ln \lambda\left(\Delta_{n}(x)\right)}=\frac{1}{2}, \forall x \in T .
$$

Using Theorem 2.5 from [16] and the faithfulness of $\mathcal{A}$ we get

$$
\operatorname{dim}_{H}(T, \mathcal{A})=\operatorname{dim}_{H}(T)=\frac{1}{2} .
$$

For a given $m \in \mathbb{N}$ let us consider $2^{m}$ probability measures $\mu^{j}, j=\overline{0,2^{m}-1}$ corresponding to the random variables

$$
\xi^{j}=\sum_{k=1}^{\infty} \frac{\xi_{k}^{j}}{\prod_{i=1}^{k} 4^{i}},
$$

whose independent digits $\xi_{k}^{j}$ have the following distributions:

if $k \neq 2^{s}$, then $\xi_{k}^{j}$ takes values $0,1, \ldots, 2^{k}-1$ with probabilities $\frac{1}{2^{k}}$;

if $k=2^{s}, s \neq m$, then $\xi_{k}^{j}$ takes values $0,1, \ldots, k \cdot 2^{k}-1$ with probabilities $\frac{1}{k \cdot 2^{k}}$;

if $k=2^{m}$, then $\xi_{k}^{j}$ takes values $j \cdot 2^{k}+0, j \cdot 2^{k}+1, \ldots,(j+1) \cdot 2^{k}-1$ with probabilities $\frac{1}{2^{k}}$.

Let $S_{j}$ be the spectrum of the measure $\mu^{j}$. From the construction of these measures and the definition of the set $T$ it follows that $S_{j} \bigcap S_{i}=\emptyset$ and $T=\bigcup_{i=1}^{2^{m}} S_{i}$. Taking into account inequality $\frac{\ln \mu^{j}\left(\Delta_{n}(x)\right)}{\ln \lambda\left(\Delta_{n}(x)\right)} \geq \frac{1}{2}, \forall x \in S_{j}$, and applying the mass distribution principle simultaneously for all measures $m^{j}$, we get $H^{\frac{1}{2}}(T, \mathcal{A}) \geq 2^{m}$. Since $m \in \mathbb{N}$ can be chosen arbitrarily, we have a desired conclusion about infiniteness of $H^{\frac{1}{2}}(T, \mathcal{A})$.

On the other hand the set $T$ can be covered by $2^{1} \cdot 2^{2} \cdot \ldots \cdot 2^{2^{s}-1} \cdot 2^{1} \cdot 2^{2} \ldots 2^{s-1} \cdot 1=$ $2^{\frac{\left(2^{s}-1\right) 2^{s}}{2}+\frac{(s-1) s}{2}}$ intervals, each of them is a union of $2^{s} 2^{2^{s}}$ cylinders from $\mathcal{A}_{2^{s}}$ with length $\left(\frac{1}{4}\right)^{\frac{2^{s}\left(2^{s}-1\right)}{2}} \cdot \frac{2^{s} 2^{2^{s}}}{4^{2^{s}}}=\left(\frac{1}{2}\right)^{2^{2 s}-s}$. The $\frac{1}{2}$-volume of this covering is equal to

$$
2^{\frac{\left(2^{s}-1\right) 2^{s}}{2}+\frac{(s-1) s}{2}}\left(2^{-2^{2 s}+s}\right)^{\frac{1}{2}}=2^{-\frac{1}{2}\left(2^{s}-s^{2}\right)} \rightarrow 0, \quad(s \rightarrow \infty) .
$$

Therefore, $H^{\frac{1}{2}}(T)=0$.

By using the same techniques it is not hard to prove the following result.

Proposition 3.2. Let $n_{k}=4^{k}$ and let $\mathcal{A}$ be the corresponding faithful net-covering family generated by the Cantor series expansion. Then for any $\alpha \in(0,1)$ there exists a set $T_{\alpha}$ such that 
1) $\operatorname{dim}_{H} T_{\alpha}=\alpha$

2) $H^{\alpha}\left(T_{\alpha}, \mathcal{A}\right)=+\infty$;

3) $H^{\alpha}\left(T_{\alpha}\right)=0$.

Proof. If $\alpha=\frac{p}{q} \in(0,1)$ is a rational number, then the proof is completely similar to those in example 2 , but in the definition of the set $V_{k}$ of digits which are admissible at the k-th step of construction of $T_{\alpha}$, we define $V_{k}$ to be $\left\{0,1, \ldots,\left[\left(n_{k}\right)^{\frac{p}{q}}\right]-1\right\}$ instead of $\left\{0,1, \ldots,\left[\left(n_{k}\right)^{\frac{1}{2}}\right]-1\right\}$ for $k \neq 2^{s}$, and $V_{k}=\left\{0,1, \ldots, k \cdot\left[\left(n_{k}\right)^{\frac{p}{q}}\right]-1\right\}$ for $k=2^{s}$, where $[\cdot]$ denotes the integer part of an argument.

If $\alpha$ is an irrational number from $(0,1)$, then we choose an increasing sequence $\left\{\frac{p_{k}}{q_{k}}\right\}$ of rational numbers converging to $\alpha$ and apply the same technics with the following definition of the set $V_{k}:=\left\{0,1, \ldots,\left[\left(n_{k}\right)^{\frac{p_{k}}{q_{k}}}\right]-1\right\}$.

Remark 3.3. The sequence $n_{k}$ has been chosen to be $\left\{4^{k}\right\}$ only for the simplicity of calculations in the above examples. In the forthcoming paper we shall show how the latter statement can be generalized and give necessary and sufficient conditions for the Cantor net-coverings to be comparable.

Remark 3.4. The latter proposition shows extreme differences between comparable and faithful net-coverings and demonstrates that the class of faithful net-coverings is essentially wider then the class of comparable ones. The relation between these two classes is similar to the relation between bi-Lipshitz transformations and transformations preserving the Hausdorff dimension (see, e.g., [7, 9] for details). More deep connections between faithfulness of net-coverings and the theory of transformations preserving the Hausdorff dimension will also be discussed in the forthcoming paper.

\section{REFERENCES}

[1] S. Albeverio, Yu. Kondratiev, R. Nikiforov, G. Torbin, On new fractal phenomena connected with infinite linear IFS, Mathematische Nachrichten, 290(8-9) (2017), P. 1163-1176.

[2] S. Albeverio, Yu. Kondratiev, R. Nikiforov, G. Torbin, On fractal properties of non-normal numbers with respect to Renyi f-expansions generated by piecewise linear functions. Bulletin des Sciences Mathematiques, 2014, 138(3), 440-455.

[3] S. Albeverio, V. Koshmanenko, M. Pratsiovytyi, G. Torbin, On fine structure of singularly continuous probability measures and random variables with independent $\widetilde{Q}$ - symbols, Methods of Functional Analysis and Topology, 17(2011), No. 2, P. 97-111.

[4] S. Albeverio, I. Garko, M. Ibragim, G. Torbin, Non-normal numbers: Full Hausdorff dimensionality vs zero dimensionality. Bulletin des Sciences Mathematiques, 141(2) (2017), P. 1-19.

[5] S. Albeverio, M. Pratsiovytyi, G. Torbin, Topological and fractal properties of real numbers which are not normal. Bulletin des Sciences Mathematiques, 2005, 129(8), 615-630.

[6] S. Albeverio, M. Pratsiovytyi, G. Torbin, Singular probability distributions and fractal properties of sets of real numbers defined by the asymptotic frequencies of their s-adic digits. Ukrainian Mathematical Journal, 2005, 57(9), 1361-1370.

[7] S. Albeverio, M. Pratsiovytyi, G. Torbin, Fractal probability distributions and transformations preserving the Hausdorff-Besicovitch dimension, Ergodic Theory and Dynamical Systems, 24(2004), No. 1, P. 1-16.

[8] S. Albeverio, O. Baranovskyi, M. Pratsiovytyi, G. Torbin, The Ostrogradsky series and related Cantor-like sets. Acta Arithmetica, 130(3)(2007), P. 215-230.

[9] S. Albeverio, M. Pratsiovytyi, G. Torbin, Transformations preserving the Hausddorff-Besicovitch dimension, Central European Journal of Mathematics, 6(2008), No. 1, P. 119-128.

[10] S. Albeverio, G. Torbin, On fine fractal properties of generalized infinite Bernoulli convolutions, Bull. Sci. Math., 132(2008), No. 8, P. 711-727.

[11] S. Albeverio, G. Torbin, Fractal properties of singularly continuous probability distributions with independent $Q^{*}$-digits, Bull. Sci. Math., 129(2005), No. 4, P. 356-367.

[12] C. Bandt, S. Graf, M. Zähle - eds, Fractal Geometry and stochastics, Basel, Boston, Berlin, Birkhäuser, (2000). 
[13] K. Baranski, Hausdorff dimension of the limit sets of some planar geometric constructions, Advances in Mathematics, 210(2007), P. 215-245.

[14] M. Bernardi, C. Bondioli, On some dimension problems for self-affine fractals, Journal for Analysis and its Applications, 18(1999), No. 3, P. 733-751.

[15] A. Besicovitch, On existence of subsets of finite measure of sets of infinite measure, Indag. Math, 14(1952), P. 339-344.

[16] P. Billingsley, Hausdorff dimension in probability theory II, Ill. J. Math., (1961), No. 5, P. 291-198.

[17] P. Billingsley, Ergodic theory and information, New York-London-Sydney: John Wiley \& Sons, Inc., (1965).

[18] G. Cantor, Über die einfachen Zahlensysteme, Zeitschrift f. Math. u. Physik., 14(1869), P. 121-128.

[19] M. Cooper, Dimension, measure and infinite Bernoulli convolutions, Math. Proc. Cambr. Phil. Soc., 124(1998), P. 135-149.

[20] C. Cutler, A note on equivalent interval covering systems for Hausdorff dimension on $R$, Internat. J. Math. and Math. Sci., 2(1988), No. 4, P. 643-650.

[21] P. Erdös, A. Renyi, Some further statistical properties of the digits in Cantor's series, Acta Math. Acad. Sci. Hungar., 10(1959), P. 207-215.

[22] K. Falconer, The geometry of fractal sets, Cambridge University Press, (2002).

[23] K. Falconer, Fractal Geometry: Mathematical Foundations and Applications, Chichester: Wiley, (1990).

[24] K. Falconer, Techniques in Fractal Geometry, Chichester: Wiley, (1997).

[25] J. Galambos, Representations of real numbers by infinite series, Lecture Notes in Mathematics,Springer-Verlag, Berlin-New York, 502(1976).

[26] I.Garko, R. Nikiforov, G. Torbin, On the G-isomorphism of probability and dimensional theories of representations of real numbers and fractal faithfulness of systems of coverings. Theory of Probability and Mathematical Statistics, 94(2017), P. 17-36.

[27] M. Ibragim, G. Torbin, A probabilistic approach to studies of DP-transformations and faithfullness of covering systems to evaluate the Hausdorff-Besicovitch dimension. Theory of Probability and Mathematical Statistics, 92(2016), P. 23-36.

[28] B. Mandelbrot, The Fractal geometry of nature, Freeman and Co, San-Francisco, (1983).

[29] B. Mance, Normal numbers with respect to the Cantor series expansion, Thesis (Ph.D.), The Ohio State University (2010).

[30] J. Marstrand, The dimension of Cartesian product sets, Proc. Cambridge Phil. Soc., 50(1954), P. 198-202.

[31] P. Mattila, Geometry of sets and measures in euclidean spaces, Cambridge University Press, (2004).

[32] R. Nikiforov, G. Torbin, Fractal properties of random variables with independent $Q_{\infty}-$ symbols. Theory of Probability and Mathematical Statistics, 86( 2013), P. 169-182.

[33] Y. Peres, W. Schlag, B. Solomyak, Sixty years of Bernoulli convolutions, Fractal geometry and stochastics II (Greifswald/Koserow, 1998), Progr. Probab., Birkhäuser, Basel, 46(2000). P. 39-65.

[34] Y. Peres, B. Solomyak, Absolute continuity of Bernoulli convolutions, a simple proof, Math. Res. Lett., 3(1996), No. 2, P. 231-239.

[35] Y. Peres, G. Torbin, Continued fractions and dimensional gaps, in preparation.

[36] Y. Pesin, Dimension theory in dynamical systems. Contemporary views and applications, Chicago Lectures in Mathematics. University of Chicago Press, Chicago, (1997).

[37] M. Pratsiovytyi, Fractal approach to investigations of singular distributions, National Pedagogical Univ., (1998).

[38] N. Pratsevityi, G. Torbin, Superfractality of the set of numbers having no frequency of n-adic digits, and fractal probability distributions. Ukrainian Mathematical Journal, 1995, 47(7), 1113-1118.

[39] M. Pratsiovytyi, G. Torbin, On analytic (symbolic) representation of one-dimensional continuous transformations preserving the Hausdorff-Besicovitch dimension, Transactions of the National Pedagogical University of Ukraine. Mathematics, 4(2003), P. 207-215.

[40] C. Rogers, Hausdorff measures, Cambridge Univ. Press, London, (1970).

[41] F. Schweiger, Ergodic Theory of Fibred Systems and Metric Number Theory, Oxford: Clarendon Press, (1995).

[42] B. Solomyak, On the random series $\sum \pm \lambda^{n}$ (an Erdös problem), Ann. of Math., 142(1995), No. 3, 611-625.

[43] G. Torbin, Multifractal analysis of singularly continuous probability measures. Ukrainian Mathematical Journal, 57(5) (2005), P. 837-857.

[44] A. Turbin, M. Pratsiovytyi, Fractal sets, functions, and distributions,. Kiev: Naukova Dumka, (1992).

[45] H. Triebel, Fractals and Spectra, Basel, Boston, Berlin, Birkhäuser, (1997). 
S. Albeverio: albeverio@uni-bonn.de

Institut für Angewandte Mathematik, Universität Bonn, Endenicher Allee 60, D-53115 Bonn (Germany); HCM and SFB 611, Bonn; BiBoS, Bielefeld-Bonn; CERFIM, Locarno; IZKS, Bonn

Ganna Ivanenko: anna.ivanenko@ukr.net

National Dragomanov Pedagogical University, Pyrogova str. 9, 01030 Kyiv (Ukraine)

Mykola LeBid: mlebid@math.uni-bielefeld.de

Fakultät für Mathematik, Universität Bielefeld, Postfach 1001 31, D-33501, Bielefeld (Germany); National Dragomanov Pedagogical University, Pyrogova str. 9, 01030 Kyiv (Ukraine)

Grygoriy Torbin: g.m.torbin@npu.edu.ua

National Dragomanov Pedagogical University, Pyrogova str. 9, 01030 Kyiv (Ukraine); Institute for Mathematics of NASU, Tereshchenkivs'ka str. 3, 01601 Kyiv (Ukraine) 